\title{
LA ANTROPOLOGÍA DE GÜNTHER ANDERS EN EL MARCO DE UNA DISONANCIA EXISTENCIAL ${ }^{1}$ (1 ${ }^{\text {a }}$ PARTE)
}

\section{THE ANTHROPOLOGY OF GÜNTHER ANDERS IN THE FRAMEWORK OF EXISTENTIAL DISSONANCE (PART I)}

\author{
BRUNO ONETTO MuÑOZ \\ Universidad Austral de Chile - Valdivia
}

RESUMEN: El siguiente texto busca acercar la figura de Gunther Anders al contexto filosófico europeo de su época, poniendo de relieve los temas desarrollados por este escritor-filósofo así como la visión antropológica-filosófica por él desarrollada, y en contraste con la filosofía existencial heideggeriana de la época.

Palabras Clave: Antropología filosófica, antropología negativa, existencia, vida, extrañeza del mundo.

ABSTRACT: The following text seeks to show the present-day relevance of Günther Anders' philosophy, which includes not only an initial philosophical anthropology, but a technical philosophy, both of which provide a contrast to Heidegger's philosophy.

KEYWORDS: Philosophical anthropology, negative anthropology, existence, life, world strangeness.

${ }^{1}$ El siguiente texto forma parte de una investigación inicial sobre la relevancia del pensamiento de Günther Anders, en la actual época de los medios masivos de la comunicación. A esta indagación ha de seguirle el inicio de una crítica radical desde esta situación parcial y negativa del hombre en el mundo. El Proyecto de investigación científica fue iniciado el año 2010 para el Fondo Nacional de Ciencia y Tecnología, en Chile (Fondecyt \#1100694, 2010/13). 


\section{Introducción}

Si quisiéramos responder a la pregunta de en qué cifrar la vigencia de un autor como Günther Anders, podríamos mencionar como evidencia aquel reciente y ya pasado anuncio de unas primeras jornadas andersianas, las últimas que han buscado revisitar la actualidad de su pensamiento para el medio académico alemán, durante el año recién pasado, en la Facultad de Filosofía de Marburgo (Zur Aktualität Günther Anders, 14-15 mayo, de 2010)²; o referirnos a la más reciente biografía del autor publicada por Raimund Bahr, Leben und Denken im Wort (Edition-Art-Science, Wien 2010) o incluso siendo aún más enfáticos: destacar la publicación, en fecha reciente y hace tiempo esperada por el lector de habla castellana, de los dos volúmenes de su principal obra, «La obsolescencia del hombre» (Die Antiquierheit des Menschen, 2 vols. 1954/1980) (AM), por la editorial española Pre-textos (2011). Lo que en definitiva no es más que un síntoma, que no viene a disminuir en nada el hecho de que el estado de reflexión de los temas abordados por este ensayista sea casi un desideratum forzoso para la actualidad de la filosofía de la técnica en nuestra época, por ser Anders quizá el único (si no el primero, tal vez), en llevar a cabo un real enfrentamiento con el mundo técnico de los actuales medios de comunicación, a mediados de los cincuenta y con la era atómica. Sin dejar de inquirir jamás, con su amplificada crítica, en la difícil relación de ser humano con su producto más relevante y peligroso, como ha demostrado ser durante todo el siglo pasado o por doquiera el mundo de la técnica. Este mundo y no otro, es el que en su imparable expansión y de forma casi irreversible, parece estar haciendo desaparecer nuestra genuina condición humana convirtiéndola en parte integrante y funcional de aquella sociedad globalizada y tecnificada que nos toca vivir.

De allí que indagar por la definición de la naturaleza del hombre en contraposición con la del animal o la de los otros entes, en general, mucho más contingentes y reemplazables (si se atiende como la época tan sólo a su utilidad), puede resultar hoy una tarea «filosóficamente infantil» (AM2: 129), como igual-

${ }^{2}$ Cfr. http://www.friedensnews.at/?s=g\%C3\%BCnther+Anders, jornadas en las que participaran Christian Dries, Stefan Broniowski, Paola Bozzi, y Raimund Bahr, cfr. en: http://www.friedensnews.at/2010/05/02/die-welt-als-vernichtungslager/\#more-6809; por la reciente biografía cfr: http://www.editionas.net/sites/buch_andersbio.html (Consultado el 2 de febrero, 2011). 
mente no aceptada por algunos (¿qué más habría de aclarar esa irreducible nota de la «diferencia específica» para nuestra presunta determinación humana, en una época en que no es el mundo animal quien se va a configurar como plano de fondo de nuestra existencia sino un mundo fabricado y lleno de productos y aparatos? AM1: 327). Pero aquello no fue óbice alguno para que al inicio del siglo $\mathrm{XX}$, y testimoniado por una fuerte necesidad de una reflexión que afrontase aquel sentimiento oscuro y de horror, producido por la Gran Guerra, naciera una escuela de corte más legítimo, que rescatase lo fáctico y existencial (Wittulski: 1992, 22). Un pensamiento mucho más situado en términos históricos y sociales, porque reflejaba de una forma no intencionada, y especulativamente, la situación social efectiva o concreta de la posguerra: donde «la nada» existencial concreta en la cual se encontraba aquel soldado que fuera derrotado en la guerra y que retornaba a casa, era la de un desocupado o la de un falto de hogar, convirtiéndose luego sin una explicación social e histórica satisfactoria (en Heidegger y el existencialismo, al menos) en ese anónimo «uno», y por ello, en una reflexión más próxima al ser del individuo (que del mundo), peligrosamente desvinculado y alejado de lo social (Anders: 2001, 69, 114, 147, 207), pero también - como cree Anders - distanciado de la desvanecida y algo desprestigiada realidad del siglo anterior, no por ello menos relevante: de la naturaleza. El pensamiento contemporáneo pareciera no querer abandonar ese itinerario de ruta, insistiendo en la búsqueda de nuestra adecuación con el mundo, de esa naturaleza animada del hombre, aunque no definida en su ser más esencial. Y ello no para evitar su ya evidente o probada barbarie, sino para saber si aún es posible una digna y cada vez más urgente inserción de su ser en el mundo.

Günther Anders ha permanecido como un autor y crítico pensador poco leído fuera del mundo germánico y por ende casi un desconocido en el ámbito filosófico, que se ha ocupado a lo largo del siglo XX, y en lo grueso de su pensamiento, al menos de dos grandes temas (en su singular estilo no del todo académico por el público al que iba dirigido), pero temas que le han vinculado doblemente a la obra de Martin Heidegger y metodológicamente tal vez incluso a la de su primer maestro: Edmund Husserl. Pensamientos que nos recuerdan aquellas viejas objeciones hechas a una forma de hacer filosofía que se dispensaba de antemano del compromiso inmediato con el devenir político y la historia, o al menos así lo pretendía. El primer tema de su reflexión filosófica-académica, como lo anticipara ya Heidegger, lo expone Anders igualmente como una "auto-aclaración de la existencia», como el intento de situar al 
anthropos en el mundo, y que desarrollara públicamente en sus primeros escritos editados en pleno exilio propiamente. Se trata de dos artículos editados en Francia ${ }^{3}$, en los que se indaga en una incipiente antropología filosófica de signo negativo. Una reflexión crítica que tomará distancia frente a la más «insigne» analítica existencial heideggeriana, realizando con ello una de las primeras confrontaciones críticas desde el interior de la nueva filosofía del siglo. El segundo tema lo conforma su enfrentamiento con el mundo de la técnica, y lo comienza a inscribir, con su retorno del exilio a Europa, en los 50 (conmovido, asimismo, por el crimen de Hiroshima, acaecido el 6.8.45), en su gran opera: «La obsolescencia del hombre» (Die Antiquierheit des Menschen) y le convertirá en una de las conciencias más lúcidas de la era tecnológica. No obstante, esta obra, tan poco recepcionada como traducida, en el ámbito filosófico mundial, anticipa la primera crítica antropológica a los medios masivos de comunicación, a la radio y la televisión, obra que se concretizará en una filosofía de la técnica para la era postindustrial, expresando una fuerte crítica a la sociedad y cultura tecnificada de hoy así como al despliegue inconciente del armamentismo atómico de posguerra.

Günther Stern (1902-1992), como se le conociera al filósofo antes de iniciar su carrera pública de crítico y escritor freelance, en el Börsen Courier berlinés, pertenecía - como lo destacara el fallecido filósofo Franco Volpi ${ }^{4}$ - al círculo de los más señeros discípulos judíos de Martin Heidegger (Arendt, Jonas, Löwith, Strauss). Stern había estudiado cuatro semestres lectivos en Friburgo (1923/24) y realizado ya antes de conocer a Heidegger, en Marburgo (ca. 1925), su promoción con Edmund Husserl el año 24, con una tesis sobre «El rol de la categoría de la situación en los enunciados lógicos». Su propósito en ella era indagar acerca de la posibilidad y la forma de utilizar el método fenomenológico en juicios, cuestiones y frases de la vida común y corriente, auscultando hasta dónde habría de jugar un rol decisivo en todos ellos su dependencia de la situación pragmática o concreta en la que aquellos surgen (Wittulski: 23s.).

${ }^{3}$ Une interprétation de l'a posteriori, en: Recherches Philosophiques, vol. IV, Paris 1934/5, 6580; y Pathologie de la liberté, en: Recherches Philosophiques, vol VI, Paris 1936/7, 22-54.

${ }^{4}$ Cfr. Sobre Heidegger: cinco voces judias. Günther Anders, Hannah Arendt, Hans Jonas, Karl Löwith, Leo Strauss. Introducción de Franco Volpi. Buenos Aires: Manantial, 2008. 


\section{Una antropología filosófica de corte negativa}

Contrario a lo que podría esperarse respecto de una relación cordial con su maestro, esto es, con una rápida adhesión y fidelidad del adepto con el método fenomenológico, Anders - crítico como lúcido en su reflexión- se resistió desde un comienzo a la línea fenomenológica por él elegida y de una manera radical. Según ésta, toda expresión subjetiva dicha por un yo podía ser siempre sustituida por una objetiva, suprimiendo de ese modo el contenido de la situación que la había hecho surgir. Ante lo cual Anders se opone con una decisiva objeción, porque una frase dicha por un yo, o que concierne al respectivo Da-sein, no sólo contiene datos empírico-fácticos, sino que no puede ser sustituida tampoco por otra dicha en tercera persona, sin que con ello no se pierda o abandone lo específico de la frase dicha por aquel yo. Porque el sentido del ser del habla está condicionado por la situación personal. El habla constituye él mismo una situación nueva para una persona, y ejemplos de ello lo podemos observar en la confesión, la justificación, el desahogo de los sentimientos, etc. Indagar en la frase, en el enunciado de un yo nada más que el contenido noemático de la expresión, deja fuera de juego precisamente la verdadera experiencia noemática; es imposible aislar para sí eso expresado por un yo, por lo que la frase sola no agota su contenido. En los enunciados del yo, el acto que presupone el yo no es propiamente uno teórico (Wittulski, 24). Con ello Anders ponía su índice sobre una falencia in situ al método fenomenológico mismo. La desconexión de la génesis subjetiva del acto mismo del pensamiento en el análisis del significado, debería reponer en seguida necesariamente las categorías de tiempo y realidad, pues ciertos enunciados — digamos, los más contingentes - no se podrían entender ni mucho menos construir sin aquellas. Todo conocimiento — sea todo lo abstracto que se quiera - pertenece a una situación concreta, aunque de ella no seamos concientes del todo. Lo que no les afecta en su verdad, sino en su pretensión de validez absoluta. Lo inverso es, por otro lado, la exigencia que pone Husserl: la situación no tiene importancia, puesto que es la evidencia, el «a priori», un estado de conocimiento histórico absoluto que no precisa de ningún índice temporal, y de ese modo es independiente de toda realidad. Anders da entonces sus primeros pasos fuera de la fenomenología desarrollando otro camino para su indagación sobre la realidad del hombre. Asumiendo la dimensión temporal de los fenómenos, en efecto, destaca la posibilidad de que ciertos objetos, especialmente los de fabricación técnica, invenciones como la televisión o la bomba, ten- 
gan una incidencia sobre nuestro ser transformando y suscitando también con ello secuelas de orden social imprevistas que terminan marcándonos en el transcurso del tiempo. Un camino paralelo quizá al de Heidegger pero no idéntico.

El año siguiente a la publicación de su trabajo doctoral, 1929, fue bastante crítico para Anders, pues el filósofo se hallaba ante la posibilidad del comienzo de una carrera académica, viviendo en la gran metrópolis berlinesa, en plena crisis económica-mundial, y debiendo intentar ese mismo año cumplir con su habilitación, y sin grandes perspectivas de trabajo en Berlín. Sin embargo, Anders elige hacer su habilitación en Frankfurt, en la cercanía de Adorno y Horkheimer, para quienes su procedencia, más cercana por estudios a Heidegger, resultaba sospechosa. Preparó entonces una conferencia ensayo para la Kant-Gesellschaft, que debería servir para su futura habilitación en Frankfurt, en Febrero de 1930. Y que le instalaría al menos como Privatdozent y la posibilidad de mejorar su situación económica y académica. La conferencia llevaba por título: «La extrañeza del hombre en el mundo» (Die Weltfremdheit des Menschen). Con todo, el texto leído llevaba un título diferente: "Freiheit und Erfahrung» (Libertad y experiencia). Los dos conceptos que se convertirán en el tema principal de su reflexión de entonces, pero que nominalmente dirigen su mirada hacia una disciplina filosófica que se "comprometía» con la acción y vinculaba asimismo el análisis de la experiencia existencial con el concepto de la libertad como posibilidad empírica concreta de una relación (transformadora) con el mundo de la vida.

Anders viene a desarrollar, entonces, y de esta forma, ante todo, «una antropología filosófica negativa» que se niega de antemano a entregar directrices que sean deducidas de una definición «a priori» del hombre. Definición que sólo reflejaría un antropocentrismo que el autor quiere evitar desde el inicio en su preocupación por el hombre en una época marcada tecnológicamente. Puede decirse que allí radica su principal rechazo de la analítica existencial de Sein und Zeit: en su prematura y premeditada separación que hace ésta del mundo de la naturaleza, y de idéntico modo como lo hiciera Husserl a través de la epoché fenomenológica, con su radical «apriorismo». Con ello cierra, en efecto, la analítica existencial heidedeggeriana a una verdadera concreción del ente existente ${ }^{5}$. Ya

${ }^{5}$ Véase, por ejemplo, en la obra de Martin Heidegger, Ser y Tiempo, p.75, nota 1, lo siguiente: «El apriorismo es el método de toda filosofía científica que se comprenda a sí misma». Donde el apriorismo resulta ser, en este punto, la piedra angular de la analítica existencial. 
que Anders no acepta deducir la totalidad de la vida fenoménica a partir de una esencia fija o determinada de antemano del ser del hombre; por este motivo es que su análisis de la existencia debía partir como se ve en Scheler, Plessner o Misch, de una indeterminación in situ del hombre (legado nietzscheano procedente igualmente del siglo pasado). En su conferencia alemana de Frankfurt sobre «El extrañamiento del hombre en el mundo» ${ }^{6}$, el filósofo describe este hecho como un «salirse del hombre (fuera) de lo cotidiano» (Herausfallen des Menschen aus der Alltäglichkeit), haciendo un juego evidente con aquella lectura heideggeriana de la cotidianidad, que nos sugiere una constante caída del hombre (Verfallen) en el mundo, caída mundana de la existencia, de la que propiamente debía huir el cristiano primitivo y que Heidegger concibe en esos términos, en Sein und Zeit, por la misma época. Solo que para Anders, este «salirse, escurrirse del hombre fuera de la cotidianidad» es asimismo algo de orden ontológico, porque desliga al hombre también del círculo de la naturaleza, de donde a su vez formaba parte como naturaleza nacida, y que vendrá a mostrarse recién en un momento posterior en su horizonte existencial. Lo que irá definiendo a su ser así como algo siempre ulterior, postrero (Nachträgliches), determinado siempre «a posteriori». Tal es la forma además como Anders va a darnos cuenta de la «génesis ontológica» de la libertad humana (Thomä: 2003, 391). La «situación» del hombre "en el mundo» estaría determinada por un distanciamiento a priori en el mismo mundo: distancia que separa y caracteriza mi propia extrañeza con el mundo. Distancia que constituye a su vez mi discrepancia esencial con él, no una familiaridad como pretende Heidegger. Y la libertad es la consecuencia de esta extrañeza del hombre con el mundo, pues «lo que él haga a la postre con esta indeterminación, práctica e históricamente, no puede ser en lo fundamental objeto de una determinación positiva (propuesta de antes por él mismo), ya que se sustrae además a toda especulación filosófico histórica». La antropología aquí es «negativa» justamente porque siendo el hombre un extraño, en sí mismo, un

${ }^{6}$ El inédito manuscrito fue recuperado y reconstruido por Werner Reimann (Wien 1990), albacea del Archivo de Günther Anders, en Viena; las citas parciales que haremos del manuscrito se hallan integradas en la tesis-doctoral de Christian Dries, Technik als Subjekt der Geschichte? Technik- und Gesellschaftphilosophie bei Günther Anders. Tesis para la obtención del Magíster Artium, en la Albert-Ludwigs-Universität, Freiburg WS 2003/2004. (citaremos por Ms. cuando se trate del manuscrito andersiano, o por Dries cuando se trate de la tesis de Mg.) La publicación del manuscrito está para el texto de Günther Anders, Philosophische Frühschriften. Beck-Verlag, München. 
hombre esencial—y ontológicamente «sin mundo» — como lo llamará más tarde (1984)—, no le puede ser dado su relación con el mundo, su forma de vida histórica concreta de una manera a priori (Dries: 2003/4, 21s). Su distancia para con el mundo ha de ganársela apropiándosela sucesivamente a través de la práctica de las más diversas y múltiples experiencias, por medio de su libertad, a la que se halla destinado esencialmente. Un concepto de libertad — como se veque se tornará también decisivo para la obra de Jean Paul Sartre, años después, como se lo ha de confesar el mismo Sartre a Anders (baste releer el claro dictum de «El Ser y la nada» (1943): «La existencia precede a la esencia») ${ }^{7}$.

Si no ha de irse detrás de una definición propiamente del hombre, en Anders, de una antropología en su sentido más estricto, ¿qué es lo que se plantea en su obra, propiamente? ¿La vida natural: vida humana versus vida animal o vegetal? La vida -así lo había declarado y escrito Heidegger, in extenso, en el parágrafo 10 de Sein und Zeit, "...es un modo peculiar de ser, pero en esencia sólo accesible en el Dasein. La ontología de la vida se lleva a cabo por la vía de una exégesis privativa; ella determina lo que debe ser para que pueda haber algo así como un «mero vivir». La vida no es ni un puro estar-ahí (a disposición, vorhanden) ni es tampoco un Dasein. El Dasein, por su parte, nunca puede ser ontológicamente definido como vida (ontológicamente indeterminada) y además, como algo más» (Heidegger: 1997, 75). Para Anders, la vida humana no es mero y evidente seren-el-mundo; la pregunta por «la realidad del mundo exterior» no tiene nada de creada o inventada, ni siquiera podría haber llegado a ser tal si no fuera aquella intranquilizadora pregunta que es: «un pánico filosófico» (Ms. 8; Dries: 2003/4, 21). Pero, en la medida que Anders -y así lo piensa su crítico Dieter Thomä (391), acentúa la base natural de la libertad de la vida humana (que se siente) extraña en el mundo, esto es, en su carácter de sucederse con una posterioridad, se acerca más a posiciones filosóficas contrarias, vinculadas con autores más biologicistas o empiristas como Plessner o Gehlen, por ejemplo (Thomä: 2003/4; 380 ss).

Cuando Anders le entrega más peso teórico que Heidegger al carácter natural del ser del hombre (Naturhaftigkeit des Menschen) y, a la vez, torna crítica la demanda de libertad del mismo, no vuelve a caer, por ello y de esa forma, otra

${ }^{7}$ Cfr. Günther Anders, Llámese cobardía a esa esperanza. Entrevistas y declaraciones. Prólogo de Ma. Elena Rubio. Bilbao 1995,71; AM2:130. 
vez, en el dualismo precedente del siglo 19, sino que mantiene por necesidad esa doble estrategia, para compensar en cierta forma la desaparición y pérdida de los fenómenos en cuanto tales. Estos últimos habían sido neutralizados por Heidegger al trascender y suprimir la antigua dicotomía entre «naturalismo y supranaturalismo». Afirma Anders: «Lo que la filosofía existencial» en su guerra declarada contra el naturalismo no «quiere tener por verdadero, es que el hombre se halla desprendido (abgeschnitten) efectivamente, pero también, en verdad, como un pedazo de este mundo» (Anders, 2001: 141). Pues también el animal «vive» en un mundo - afirma Anders, orientado indistintamente por los experimentos de la psicología animal y las investigaciones de biólogos y zoologos como Karl von Baer o Jakob von Uexküll, de las que se servirá Heidegger mismo sin gran problema de «inexactitud» ontológica para su curso de 1929/30 (Heidegger, GA 1929/39: 1983), Die Grundbegriffe der Metaphysik. Welt —Endlichkeit-Einsamkeit, y en los que tras realizar una larga investigación fenomenológica del temple del aburrimiento, hace un decisivo giro en su lección para recoger sin más la distinción propuesta por el zóologo Jacob von Uexküll entre el «entorno» o «mundo subjetivo interno» del animal (Umwelt o Innenwelt) y el «mundo revelado» del hombre, para distinguir así sin marcada diferencia ontológica, respectivamente, la "pobreza de mundo» que constituye al primero (al animal) frente al ser «formador de mundo» que hace al segundo (al hombre). Dato que le servirá en este tiempo, distanciado ya de la propuesta filosófica inicial hecha en «Sein und Zeit», para situar más "descentradamente» al Dasein frente a lo que pudo haberse alcanzado en el descrito modo de ser existencial del ser-en-el-mundo, logrando así dejar de manifiesto con el análisis del temple del aburrimiento, y en aquella (aparente) «falta de mundo» constitutiva para/en el Dasein, la efectiva patencia del ser en el Da-sein humano. Porque -como cree Agamben: «Quizás lo cierto sea...// que la apertura del mundo humano... sólo pueda alcanzarse mediante una operación efectuada sobre lo no-abierto del mundo animal. Y el lugar de esta operación es el aburrimiento». ${ }^{8}$ Mundo nuevamente como una apertura y trascendencia no del todo alejada de la animalidad allí presente. La «antropología negativa» depende de esta diferencia ontológica implícita y supuesta respecto al mundo, no absoluta frente a la del animal (Reimann: 1990, 23s.): diferencia que declara una discrepancia (diferencia) esencial con el mundo en relación a la congruencia total del animal con su entorno; diferencia que se presenta asimismo

\footnotetext{
${ }^{8}$ Giorgio Agamben, Lo abierto. El hombre y el animal. Pre-textos, Barcelona 2005, 82, 86, 89.
} 
como insuficiente para Anders si se afirma, como lo reitera Scheler, que ella reside en la inteligencia del hombre, o sea, en lo superior suyo frente al instinto de la especie animal. Lo abierto del hombre hacia otros mundos que el mero entorno sería aquello en lo que pretenden coincidir esas miradas antropológicas. La posición central del hombre en el mundo es la que, en parte, quedaría, resuelta en aquella lección de Heidegger, toda vez que dejaría claro cómo ha de fundarse una verdadera antropología filosófica que fundamente esa posición preeminente del hombre desde la cuestión de fondo de la filosofía, a saber, desde la cuestión del ser e indique al Dasein del hombre la forma correcta de proceder desde el ser, como único responsable y fundador de aquella y las otras ontologías (Heidegger: 1929). Sin embargo, lo fundamental para llevar a cabo esa ontología del hombre, en Anders, está en no olvidar el mundo, que para el trabajo de la analítica existencial sería lo primero en caer y ser absorbido por el Dasein para su modo de ser, en cuanto ser-en y de ese modo y como ser en el mundo.

Anders sin haber podido asistir a esa lección —biográficamente tenemos que presumirlo así- observa también que el animal vive "ya siempre en un mundo», y quizás mucho más «en» su mundo que el mismo hombre. Sólo que su «estar-dentro» (Darinnensein), su «interior-categorial» es para este coetáneo primer detractor de Heidegger igualmente un planteamiento o una determinación insuficiente. Tanto como el «no-estar-dentro» del «mundo exterior» que supone la «distancia» frente al mundo. Porque no basta suplir el problema con el «a priori» del "estar ya dentro de un mundo», ni con el opuesto: del ser "para si» que luego ha de asumir el mundo y su experiencia. La propuesta de Günther Anders es la de evitar ambas posiciones: por un lado, tanto la distancia causada por una separación absoluta de «un ser sí mismo» como de otra parte la postura del «serdentro» (Darinnensein), reflejada en un enfático «ser-con» (Mit-sein), en diálogo critico dirigido por cierto a Sein und Zeit. Y tratando, así, de salvar ese abismo, unificando ambas posiciones es que Anders plantea algo que va a describir como (un) «distanciarse del hombre del mundo en el mundo» ("Abstand des Menschen von der Welt in der Welt») o bien «el ser-en como ser con distancia» ("Das Insein als Insein in Distanz»). Una combinación de este tipo es "categorialmente posible», afirma Anders, si se piensa en el concepto de potencia como lo ha expuesto Schelling en su investigación dialéctica sobre lo «condicionado e incondicionado» o acerca de «la oscuridad» y de "la luz» en su pensamiento especulativo (cfr. F. W. Schelling: Sobre la esencia libertad humana, 1809) (Ms.6-7, 9) 
Dice Anders, en su alocución de 1939: «Para que algo condicionado exista como tal —argumenta Schelling — tiene que ser un «algo mismo» (ein "es selbst» sein), porque si no lo condicionante desaparecería en lo condicionado; esto es, tendría que ser incondicionado en cierta potencia. De manera que: para que algo este inserto en el mundo, ha de estar inserto como un «algo mismo", es decir, tener su propio relieve, a saber, no surgir de un estar inserto en algo otro. Es así como el hombre, en verdad, es en el mundo, esto es, como él mismo es mundo; aunque, por otra parte, se halle separado o sustraído (enthoben) del mundo de un modo harto peculiar; teniendo que experimentarlo algo después; teniendo que interpelarlo, en efecto, recién en el logos; (pues) no lo puede anticipar materialiter; desprecia su factibilidad como siendo contingente, un mero factum, como siendo únicamente empírica, la de «este mundo»; superando el mundo que ha de hallar y con el que ha de toparse en su invención; en cuanto realizador de éste, se halla remitido en tal grado a su realidad, tan libre de ella, que su determinación del estar-ya-dentro de aquel, como mera determinación indicadora formal ya no le basta. El problema de la libertad es el motivo fundamental no expresado que se halla a las espaldas del así llamado problema del mundo exterior» (Ms.9)

El hecho de que el ente-hombre se encuentre inserto (eingebettet) aunque en cierto modo también en su condición «recortado (o separado)» del ente en su totalidad, le entrega a su ser —o hace que éste «tenga», por así decir — una cierta relativa autonomía. Ser-libre significa que se es relativo, incierto, no necesariamente en sentido trascendente ni moral, porque se nos muestra tan solo el estado parcial de integración del hombre en el mundo. La libertad del hombre es esta relativa autonomía dada por el distanciamiento (o su no adecuarse) con el mundo; «separado» de él, se pone frente a él libre. Aquello en que para la naturaleza aturdida y atrapada del animal consiste su mundo o entorno, se traduce para el ser del hombre en el hecho de su devenir individuo, o mejor dicho: su ser «dividido o dividuum», escindido como lo está por una integración parcial porque distanciada el mundo (als In-sein in Distanz). Anders piensa por eso al individuum no como uno que se constituye a sí mismo - como resultado directo de la modernidad - sino como uno des-ajado o arrojado fuera del mundo, no integrado sino des-prendido o abstraído del mundo. Esto es lo que dice: «el hecho de que un ser finito (el hombre), tenga su ser estando separado (abgeschnitten) del ser en su totalidad, y lo tenga en una cierta relativa independencia» (Anders, AM1: loc. cit., Reimann: Verweigerte Versöhung: 85; Kontrovers 59). Independencia que significa, en efecto, que: el hombre posee su libertad en forma relativa, o lo que es lo 
mismo: que el término libertad contiene en sí dos sentidos (los que en Anders se deben una vez más a la influencia de la filosofía de Schelling, en sus respuestas a Fichte). Dualidad consistente en que 1) la libertad nos dice de lo libre (de aquello que es libre), su posibilidad de ser. Nos dice algo también de aquello desde dónde es libre el sujeto. Puesto que se confirma algo desde el ente en su totalidad, desde el que se desprende o recorta el hombre. Y lo dicho es que este ente no está subordinado a aquel en su llamado, sino que dispone de un espacio de juego para su propia vida. O sea: que el problema de la libertad apunta directo a la «extrañeza del hombre en el mundo», a su estar separado de él y no a una decisión moral de un sujeto; apunta a un pararse del ser del hombre sobre sí mismo, y no a una autodeterminación moral (Ms. 11).

De esta no-identidad esencial con el mundo y no puramente fenoménica (como podría objetarse desde una analítica del tipo heideggeriano del Dasein); de esta división ingénita — pues crece desde el ser nacido (natum esse; AM1: 24) del ser humano- deviene luego para Anders la individuación: de una diferencia ontológica entre hombre y mundo. Y así también es como de esa determinada indeterminación de ser del hombre, de lo manco o deficiente de su indefinición resulta su libertad. Libertad que no ha de ser pensada de la manera hasta ahora habitual: como libre arbitrio o autonomía positiva del hombre, como una categoría moral, sino como la consecuencia necesaria del «extrañamiento del hombre en el mundo». Su antropología filosófica se entenderá, de esa manera, como «negativa», porque ha terminado de existir allí donde recién comenzaba a desenvolverse; nada del hombre ha de decirse «a priori» de la experiencia y de su libertad, sino que las ha de asumir, para llegar a ser él mismo en el mundo (Ms. 10).

El hombre se halla de esa manera inserto en el mundo, pero a diferencia del material a priori del mundo que el animal presenta natural- e instintivamente implantado en él y que le da como única posibilidad a de ser estar encerrado o sumido casi por completo y sensorialmente en él, el hombre está puesto de tal modo en su mundo que sólo ha de llegar a éste recién postreramente. Debe venir al mundo, recoger el mundo que ya se le ha anticipado en un salto de antes a él mismo. (Er muss die Welt einholen, die je schon einen Vorsprung vor ihm hat). Esta falla de ensamble, de inserción o de implante del hombre en el mundo, es esencial. Pues indica que su conocimiento es, como toda experiencia suya, a posteriori (a posteriorisch) -dicho kantianamente. El entorno y mundo del animal, no necesita una búsqueda especial, pues todo su mundo se le ha dado de antemano 
al animal. Pero ese «material a priori» del animal, la demanda «instintiva» suya si se quiere, la tiene ya cubierta del todo por el mundo que el animal puede alcanzar o al que éste se entrega desde su nacimiento, aunque no siempre lo encuentre a su disposición; de allí dos cosas: la primera, que la naturaleza instintiva mantiene (de este modo) un vínculo firme con el mundo (Das Instinktwesen hat feste Weltbindung,... Ms.6) y, por ello, puede decirse que el animal «no viene al mundo» (nicht kommt zur Welt) como nuestra humana existencia, sino que «su mundo viene con él» («seine Welt kommt mit ihm») (Ms.2/3), o que está implantado o inserto (eingepflanzt) en el mundo.

En este punto podemos decir justamente que toda experiencia para el hombre $-\mathrm{y}$ cada experiencia representa ya una comunicación del hombre con el mundo (Ms. 12) — viene a darse desde el comienzo a partir de esta situación específica del hombre extraño al mundo, situación en la que se encuentra puesto de tal forma en el mundo, que sólo «postreramente» ha de venir a éste. Situación que tomada desde una ontología hecha desde esta única y plena contingencia, nos indica que la comunicación con el mundo, aquella que hace presente nuestra experiencia con el mundo, debe contar desde un comienzo con el seren-el-mundo, a la vez que con el ser-libre-del mundo (In-der-Welt-sein; Freivon-Welt-sein). La razón de ello es que: el hombre precisa de la experiencia porque se halla cerrado (abgesperrt) al mundo, abstraído o distanciado del mismo; pero también, el cierre del hombre es soportable puesto que el hombre puede revisarlo a éste posteriormente, porque puede tener experiencias. Pero éstas no le vienen con el mundo posteriormente, sino que: el hombre, es desde sí mismo, alguien que en el transcurso de su propia vida, puede asumir y asumirá relaciones con el mundo. Pues no está destinado a priori a concebirse desde determinados materiales, de los que ya se halla libre, si bien no puede tocar nada anticipadamente. Su a priori es formal del todo, pero es el a priori de la aposterioridad que para él llega conforme a su esencia (Ms12/13).

El mundo en tanto obstáculo, como algo que esta puesto ahí adelante (Gegenüberstand) es mucho más que un índice de conocimiento teórico, sino un índice de posición teórica: esto es, una expresión que refiere la situación del hombre, que equipara en la misma posición el ser-en con el estar-fuera-de; es una expresión que denota la libertad humana para con el mundo en el mundo; y ello explica también — piensa Anders- que el modelo de experiencia del ser occidental sea el óptico, por ser el sentido de distanciamiento en que se halla el hombre en el mun- 
do, distancia que él afirma y localiza en el campo exterior aquello que se halla puesto ahí delante del hombre, destacando un allí de lo visto como un aquí del que lo ve. Ningún otro sentido puede distinguir esa distancia o separar claramente su objeto allí a distancia (Ms.13s). Descubrir ese mundo como algo no mío, sólo es posible si el mundo para el hombre le aparece siendo algo extraño, en tanto sea libre de él, siendo su relación teórica con él esa distancia relativa al mundo, así como su relación practica la acción, en donde ambos elementos son de igual prioridad en la constitución de los índices de la libertad humana.

La libertad humana constituye el inicio de la segunda parte de este texto, acerca de la instalada actualidad de esta antropología negativa, que nos obliga como seres humanos a salir al mundo para hacernos con él, apropiándolo para poder llegar a ser nosotros mismos; de allí que para Anders éste sea siempre algo artificial, en constante devenir y nunca predecible del todo: «la artificialidad es la naturaleza del ser humano y su esencia, la inestabilidad»?.

\section{Agradecimientos}

El investigador ha visitado recientemente el Archivo de G. Anders en la Österreichische-NationalBibliothek de Viena y agradece a la Sra. Andrea Hipfinger, secretaria del Literatur-Archiv, toda la ayuda prestada, así como la enorme generosidad entregada por su albacea vitalicio, Gerhard Oberschilck, editor de la obra de Anders para la Editorial Beck, en München.

\section{Bibliografía}

ANDERS, Günther:

- (2011). La obsolescencia del hombre. 2 vols., Pre-textos Valencia.

- (1956/1980). Die Antiquierheit des Menschen. 2 vols., München.

- (2007). Filosofía de la Situación. Edición de César de Vicente Hernando. Madrid.

\footnotetext{
${ }^{9}$ Günther Anders, Filosofía de la Situación. Edición de César de Vicente Hernando. Madrid 2007, 12 .
} 
- (2001). Über Heidegger. Editado por Gerdhard Oberschlick, con un prólogo de Dieter Thomä. Beck-München.

- (1995). Llámese cobardía a esa esperanza. Entrevistas y declaraciones. Prólogo de Ma. Elena Rubio. Bilbao.

- (1928). Über das Haben. Sieben Kapitel Zur Ontologie der Erkenntnis. CohenBonn.

Agamben, G. (2005). Lo abierto. El hombre y el animal. Pre-textos, Barcelona.

DrIes, C. (2003/2004). Technik als Subjekt der Geschichte? Technik- und Gesellschaftphilosophie bei Günther Anders. Tesis del Magister Artium, de la Albert-Ludwigs-Universität, Freiburg. (http://www.freidok.uni-freiburg.de/volltexte/1382/pdf/Technik_als_Subjekt.pdf).

Reimann, W. (1990). Verweigerte Versöhnung. Zur Philosophie von G. Anders. Wien.

- (1992). Nihilismus und Scham; en: Günther Anders kontrovers, editado por Konrad Paul Liessmann, München 57-71.

SCHUBERT, E., ed. (1987). Günther Anders Antwortet. Interviews \& Erklärungen.Edition Tiamat, Berlin.

Tномё, D., ed. (2003). Heidegger Handbuch. Leben-Werk-Wirkung. Verlag J.B.

Recibido: 14/03/2011

Aceptado: 7/04/2011 
